\title{
Syntheses of (+)-Alismoxide and (+)-4-epi-Alismoxide
}

Gonzalo Blay, Begoña Garcia, Eva Molina and José R. Pedro*

Departament de Quimica Orgánica, Facultat de Quìmica, Universitat de València, Dr. Moliner 50, E-46100, València, Spain

\section{SUPPORTING INFORMATION}

\section{Table of Contents}

General Experimental Procedures ...................................................SI-2

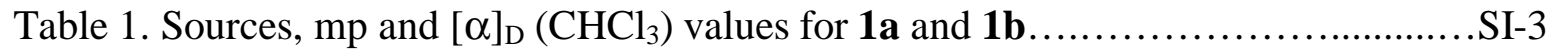

Table 2. Selected ${ }^{1} \mathrm{H}$ and ${ }^{13} \mathrm{C}$ NMR Data for compounds $\mathbf{1 a}$ and $\mathbf{1 b}$......................

${ }^{1} \mathrm{H}$ and ${ }^{13} \mathrm{C}$ NMR spectra of compound 6 .......................................................SI-5

${ }^{1} \mathrm{H}$ and ${ }^{13} \mathrm{C}$ NMR spectra of compound 7 ..............................................SI-6

NOE Experiments of compound 7 ..................................................SI-7

${ }^{1} \mathrm{H}$ and ${ }^{13} \mathrm{C}$ NMR spectra of compound 12 ..............................................SI-8

${ }^{1} \mathrm{H}$ and ${ }^{13} \mathrm{C}$ NMR spectra of compound 13 .....................................................SI-9

${ }^{1} \mathrm{H}$ and ${ }^{13} \mathrm{C}$ NMR spectra of compound 14 .................................................SI-10

${ }^{1} \mathrm{H}$ and ${ }^{13} \mathrm{C}$ NMR spectra of compound (+)-1a .......................................SI-11

${ }^{1} \mathrm{H}$ and ${ }^{13} \mathrm{C}$ NMR spectra of compound 15 ....................................................SI-12

NOE Experiments of compound 15 ..................................................SI-13

${ }^{1} \mathrm{H}$ and ${ }^{13} \mathrm{C}$ NMR spectra of compound 16 ...................................................SI-14

${ }^{1} \mathrm{H}$ and ${ }^{13} \mathrm{C}$ NMR spectra of compound (+)-1b ...........................................SI-15

COSY spectra of compound $(+)-\mathbf{1 b}$.................................................SI-16

HMQC spectra of compound (+)-1b ..............................................SI-17

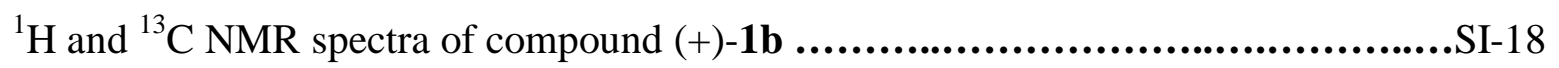

NOE Experiments of compound (+)-1b ................................................SI-19 
General Experimental Procedures. All reactions involving air or moisture sensitive materials were carried out under argon atmosphere. Commercial reagents and solvents were analytical grade or were purified by standard procedures prior to use. Compound $\mathbf{7}$ was obtained following the procedure described by us. ${ }^{1}$ Reactions were monitored by TLC analysis and the compounds purified by flash column chromatography. Melting points are uncorrected. Specific optical rotations were measured in $\mathrm{CHCl}_{3}$ using sodium light (D line $589 \mathrm{~nm}$ ). IR spectra were recorded as liquid films in $\mathrm{NaCl}$ for oils and as $\mathrm{KBr}$ discs for solids. NMR spectra were run in $\mathrm{CDCl}_{3}$, and for compounds 6-7 the solvent was filtered through basic alumina prior to use. The spectra were recorded at 300 or $400 \mathrm{MHz}$ for ${ }^{1} \mathrm{H}$ and at 75 or $100 \mathrm{MHz}$ for ${ }^{13} \mathrm{C}$ nucleus, and referenced to the solvent as internal standard. Carbon type was assigned by DEPT experiments. Low and high resolution mass spectra were recorded by electron impact (EI) at $70 \mathrm{eV}$.

\footnotetext{
${ }^{1}$ Blay, G.; Garcia, B.; Molina, E.; Pedro, J. R. Org. Lett. 2005, 7, 3291-3293.
} 
Table 1. Sources, mp and $[\alpha]_{\mathrm{D}}\left(\mathrm{CHCl}_{3}\right)$ values for $\mathbf{1 a}$ and $\mathbf{1} \mathbf{b}^{2}$

\begin{tabular}{|c|c|c|c|}
\hline Compound 1a & $\mathrm{Mp}\left({ }^{\circ} \mathrm{C}\right)$ & {$[\alpha]_{\mathrm{D}}(c$ in parentheses $)$} & Ref \\
\hline \multirow[t]{4}{*}{ Alisma orientale $^{a}$} & Oil & $+3.1(0.63)$ & $3 a$ \\
\hline & & 0 & $3 b$ \\
\hline & $140-142$ & $+8.7(0.29)$ & $3 c$ \\
\hline & $140-142$ & $+5.2(0.5)$ & $3 d$ \\
\hline Ligularia duciformis $^{a}$ & & $-5.3(0.1)$ & $4 a$ \\
\hline Aster glehni ${ }^{a}$ & Oil & $+12.1(0.097)$ & $4 b$ \\
\hline Lemnalia africana ${ }^{b}$ & $142-144$ & $0(0.1)$ & $5 a-b$ \\
\hline Xenia sp. ${ }^{b}$ & $144-145$ & $-0.3(1.2)$ & 6 \\
\hline Sinularia gardineri ${ }^{b}$ & $143 \mathrm{dec}$ & $-3.5(0.235)$ & $7 \mathrm{a}$ \\
\hline Nephthea albida ${ }^{b}$ & $132-133$ & $-1.2(0.336, \mathrm{EtOH})$ & $7 \mathrm{~b}$ \\
\hline Synthetic $^{c}$ & $138-141$ & $+8.6(1.16)$ & \\
\hline \multicolumn{4}{|l|}{ Compound $\mathbf{1 b}$} \\
\hline Silphium perfoliatum $^{a}$ & 144 & $-2.0(0.9)$ & $8 \mathrm{a}$ \\
\hline Amoora yunnanensis ${ }^{a}$ & $90-92$ & & $8 b$ \\
\hline Synthetic $^{c}$ & $59-62$ & +39.7 (0.80) & \\
\hline
\end{tabular}

\footnotetext{
${ }^{2}$ Further sources of 1a in which no characterization data are reported. (a) Curcuma zedoaria: Matsuda, H.; Morikawa, T.; Ninomiya, K.; Yoshikawa, M. Bioorg. Med. Chem. 2001, 9, 909-916, and references cited therein. (b) Guarea kunthiana: Garcez, F. R.; Garcez, W. S.; da Silva, A. G. G.; Bazzo, R. de C.; Resende, U. M. J. Braz. Chem. Soc. 2004, 15, 767-772. (c) Schisandra plena: Li, R.-T.; Zhao, A.-H.; Sheng, Y.-H.; Na, Z.; Sun, H.-D. J. Asian Nat. Prod. Res. 2005, 7, 847-852. (d) Ziziphus jujuba; Li, L.-M.; Liao, X.; Peng, S.-L., Ding, L.-S. J. Integr. Plant Biol. 2005, 47, 494-498.

${ }^{3}$ (a) Oshima Y.; Iwakawa, T. Hikino, H. Phytochemistry 1983, 22, 183-185. (b) Yoshikawa, M.; Yamaguchi, S.; Matsuda, H.; Kohda, Y.; Ishikawa, H.; Tanaka, N.; Yamahara, J.; Murakami, N. Chem. Pharm. Bull. 1994, 42, 1813-1816, and references cited therein. (c) Nakajima, Y.; Satoh, Y.; Katsumata, M.; Tsujiyama, K.; Ida Y.; Shoji, J. Phytochemistry 1994, 36, 119-127. (d) Peng, G.-P.; Tian, G.; Huang, X.-F.; Lou, F.-C. Phytochemistry, 2003, 63, 877-881.

${ }^{4}$ (a) Gao, K.; Yang, L.; Jian, Z.-J. J. Chin. Chem. Soc. 1999, 46, 619-622. (b) Min, Y. D.; Kwon, H. C.; Choi, S. Z.; Lee, K. R. Yakhak Hoeji 2004, 48, 65-69.

${ }_{5}^{5}$ (a) Bowden, B. F.; Coll, J. C.; Mitchell, S. J.; Skelton, B. W.; White, A. H. Aust. J. Chem. 1980, 33, 27372747. (b) Bowden, B. F.; Coll, J. C.; Mitchell, S. J. Aust. J. Chem. 1980, 33, 1833-1839.

${ }^{6}$ Kitagawa, I.; Kobayashi, M.; Cui, Z.; Kiyota, Y.; Ohnishi, M. Chem. Pharm. Bull. 1986, 34, 4590-4596.

${ }^{7}$ (a) El Sayed, K.; Hamann, M. T. J. Nat. Prod. 1996, 59, 687-689. (b) Su, J.-Y.; Kuang, Y.-Y.; Zeng, L.-M. Hиахие Хиеbao (Acta Chim. Sinica) 2003, 61, 1097-1100.

${ }^{8}$ (a) Bohlmann, F.; Jakupovic, J. Phytochemistry 1979, 18. 1987-1992. (b) Luo, X.; Wu, S.; Ma, Y.; Wu, D. Zhiwu Xuebao (Acta Botanica Sinica) 2001, 43, 426-430. (c) De Almeida, R. N.; Barbosa-Filho, J. M. J. Braz. Chem. Soc. 1991, 2, 71-73.
} 


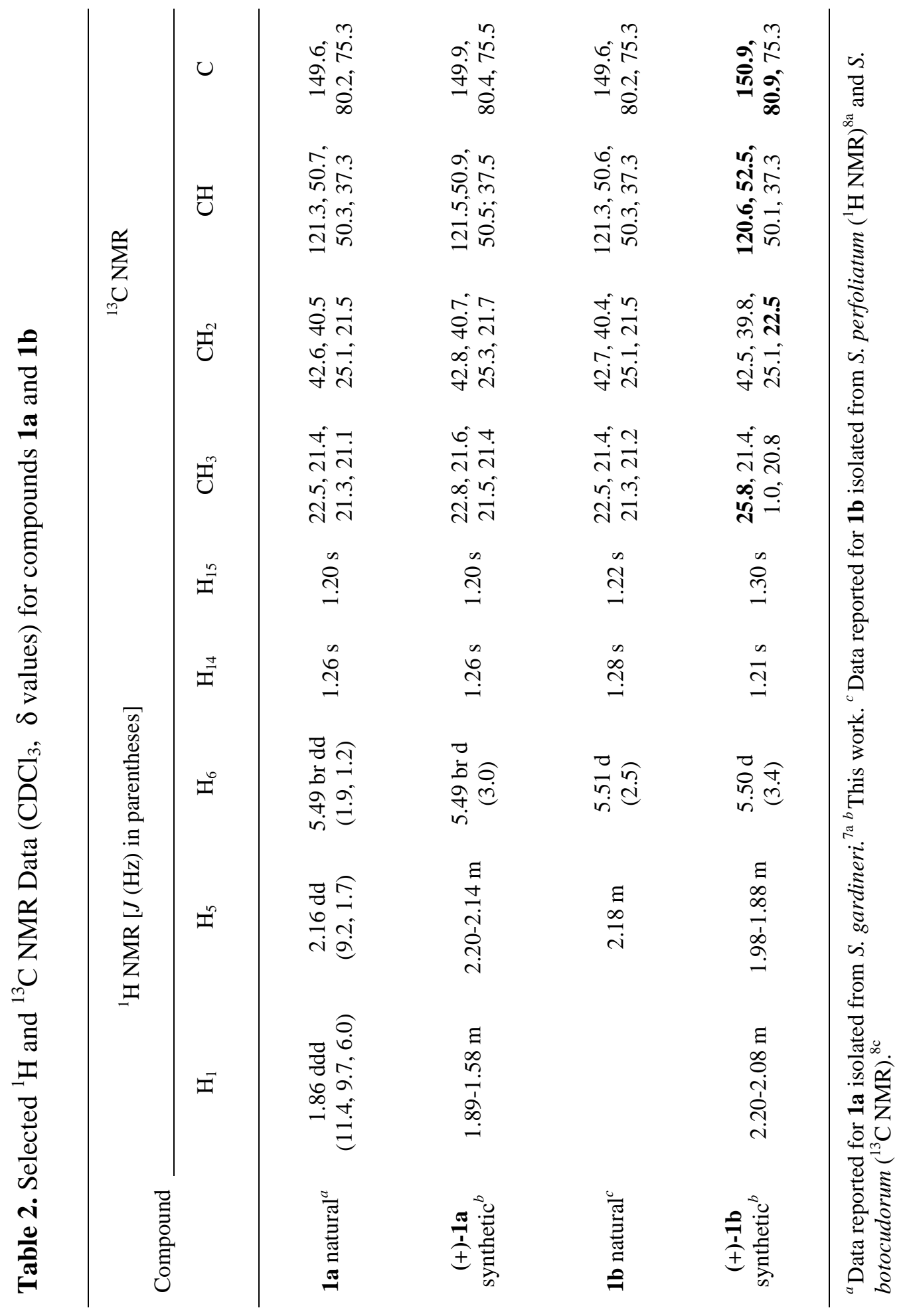




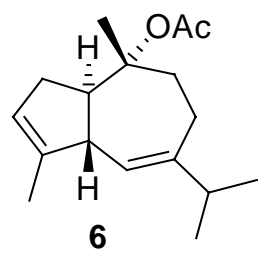

${ }^{1} \mathrm{H}$ NMR (400 MHz, $\left.\mathrm{Cl}_{3} \mathrm{CD}\right)$

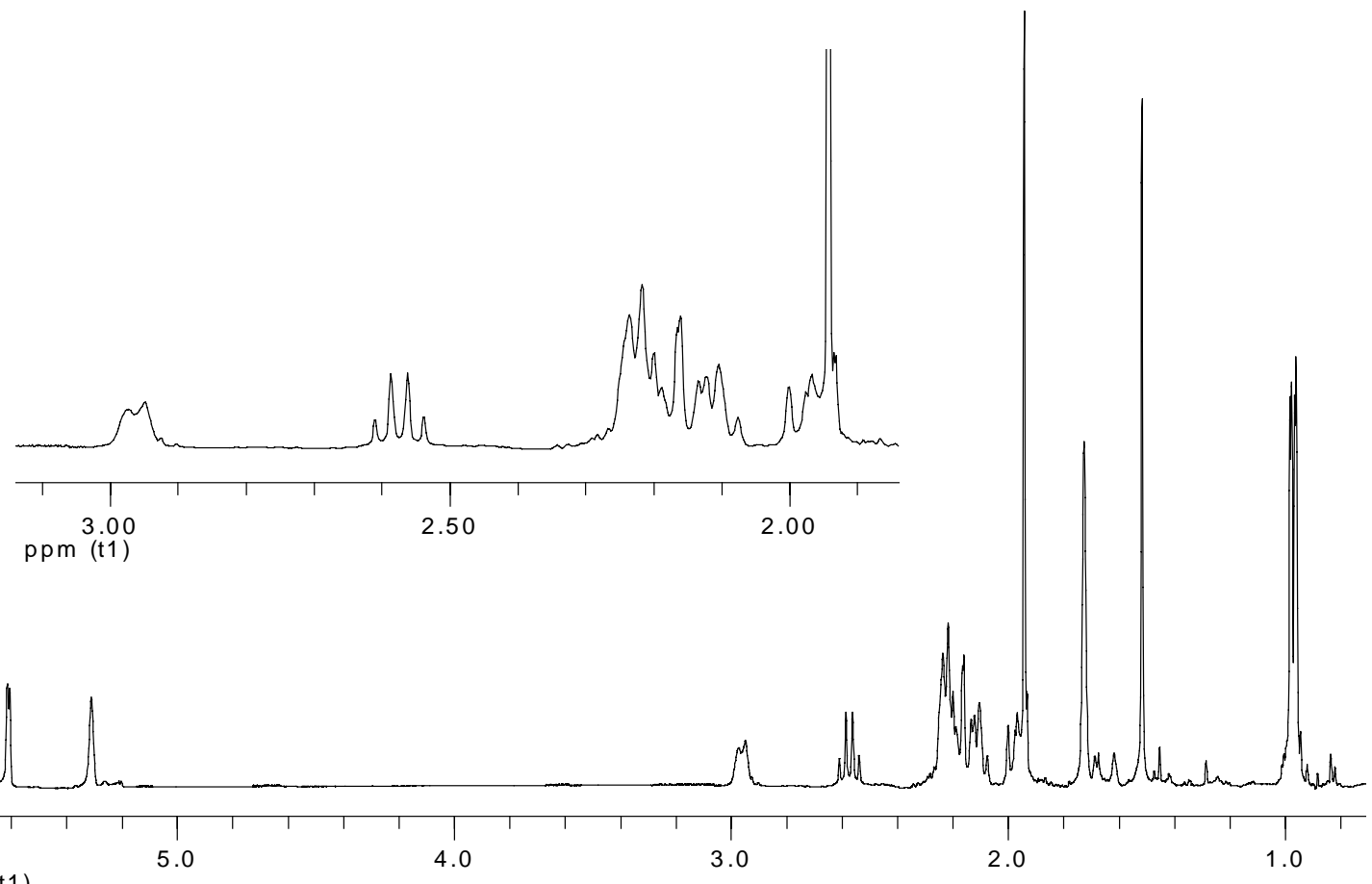

ppm (t1)

${ }^{13} \mathrm{C}$ NMR (100 MHz, $\left.\mathrm{Cl}_{3} \mathrm{CD}\right)$

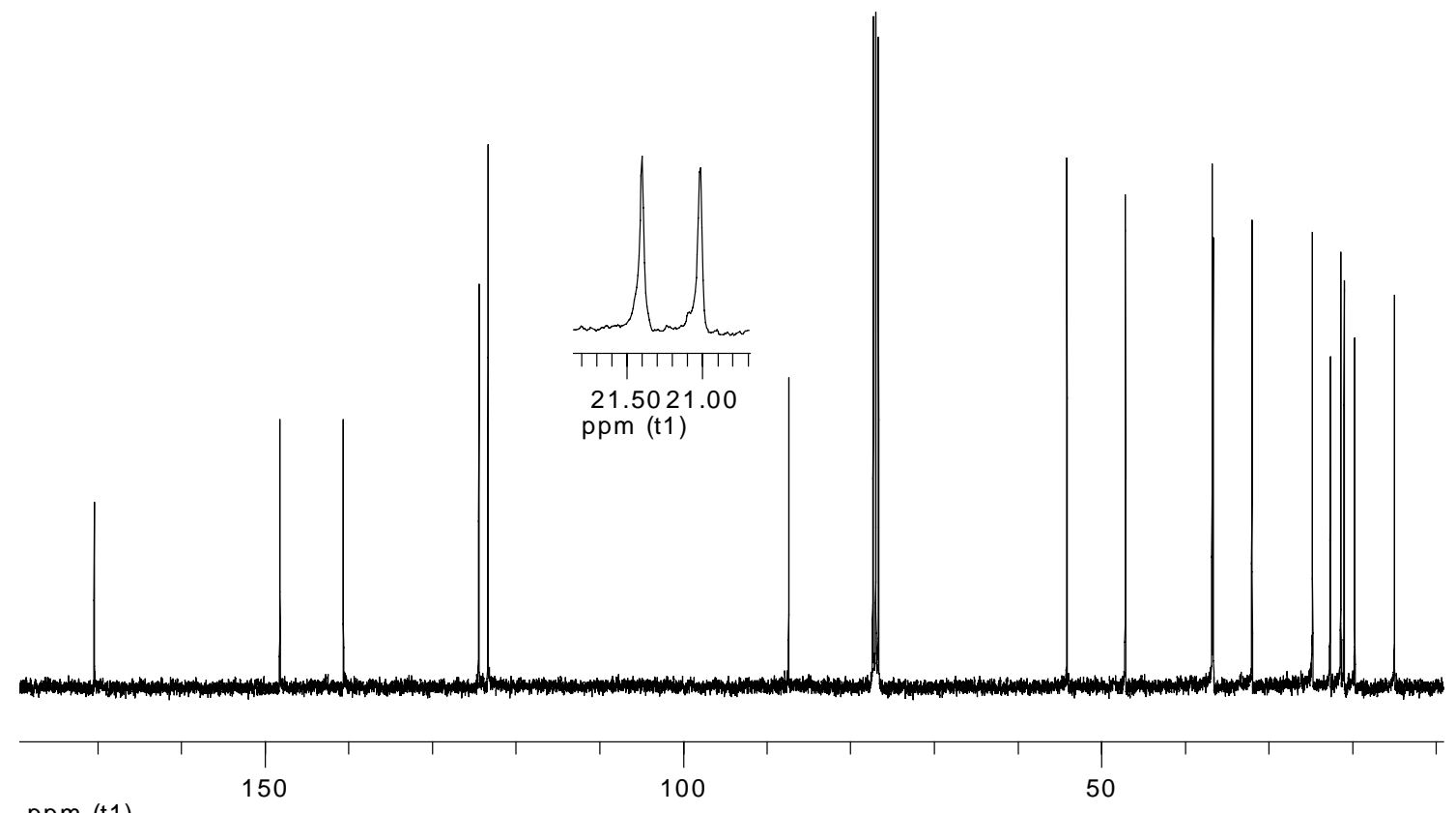

ppm (t1)

SI- 5 


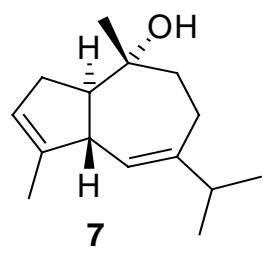

NOE Experiments (400 $\mathrm{MHz}, \mathrm{Cl}_{3} \mathrm{CD}$ )
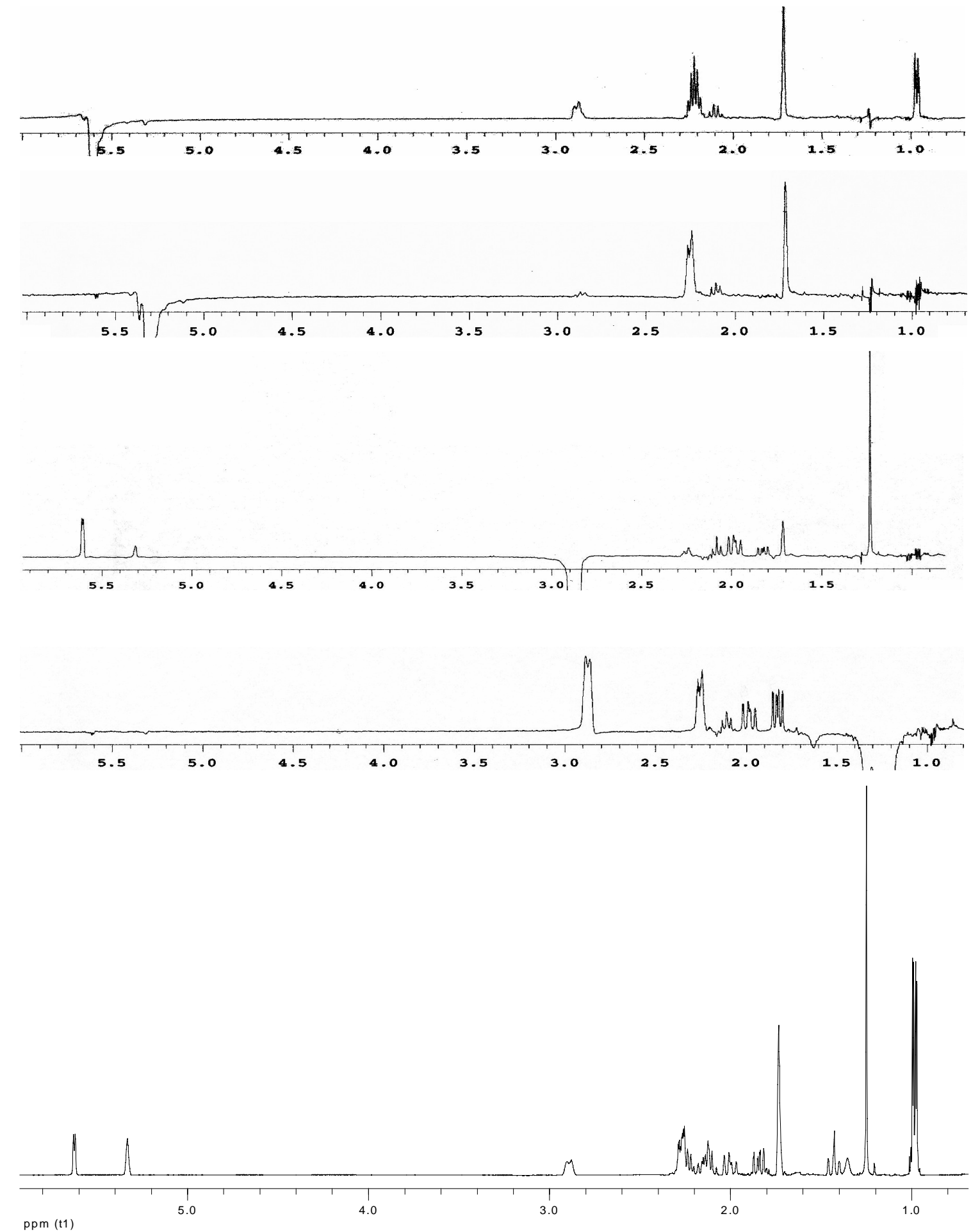

ppm (t1) 


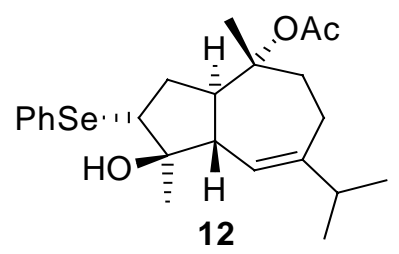

${ }^{1} \mathrm{H}$ NMR (400 MHz, $\left.\mathrm{Cl}_{3} \mathrm{CD}\right)$

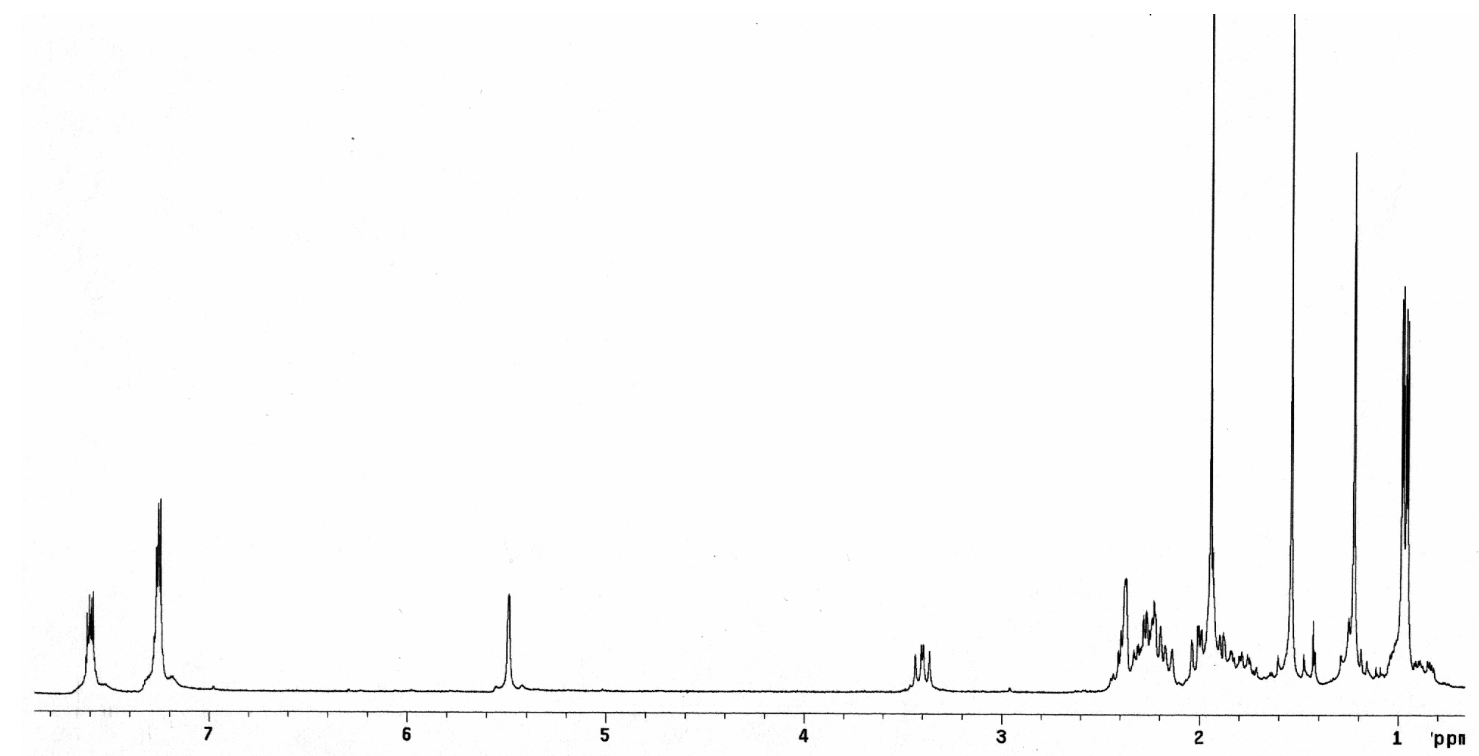

${ }^{13} \mathrm{C}$ NMR (75 MHz, $\left.\mathrm{Cl}_{3} \mathrm{CD}\right)$

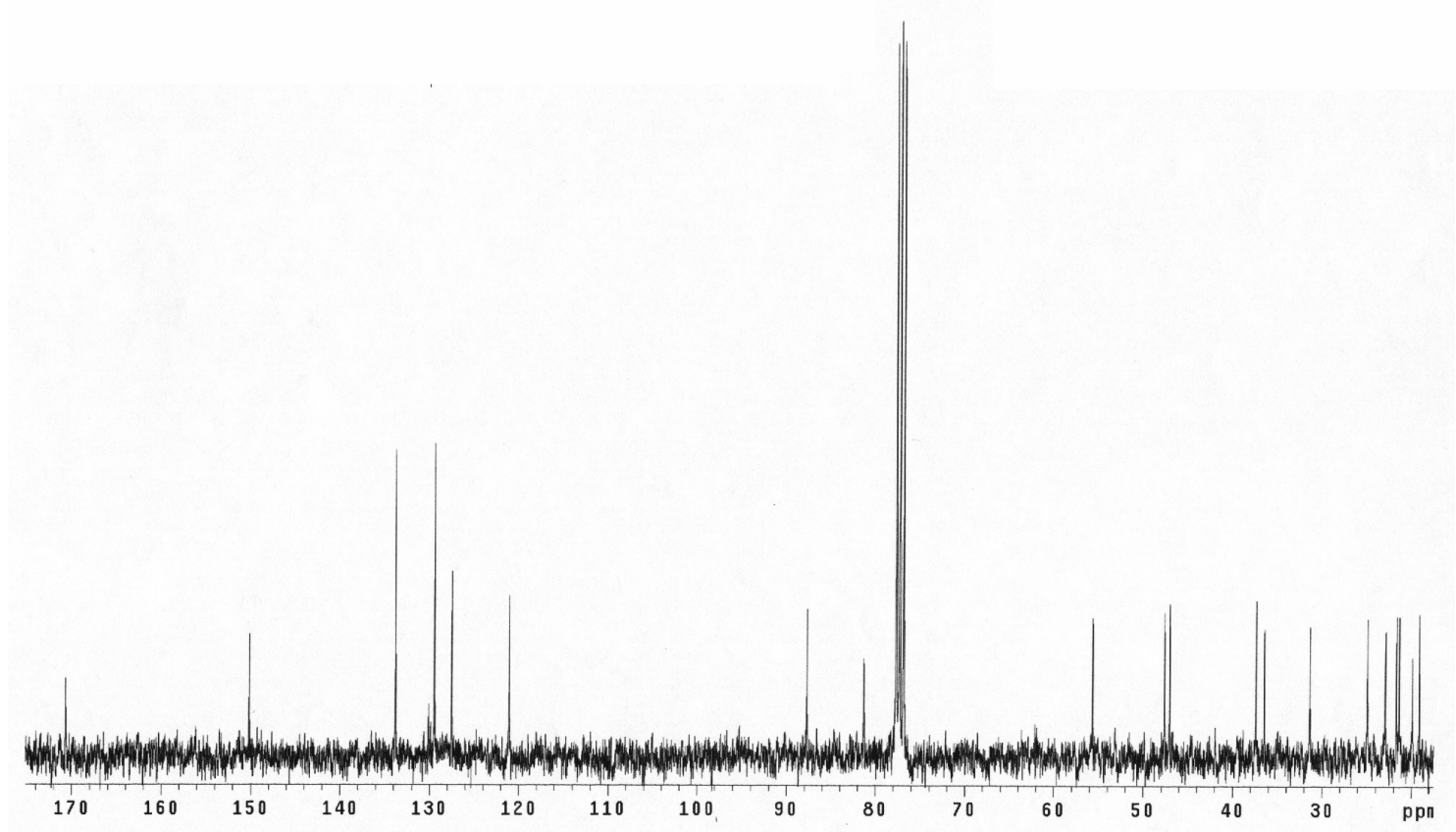




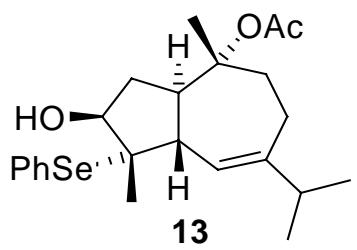

${ }^{1}$ H NMR (300 MHz, $\left.\mathrm{Cl}_{3} \mathrm{CD}\right)$

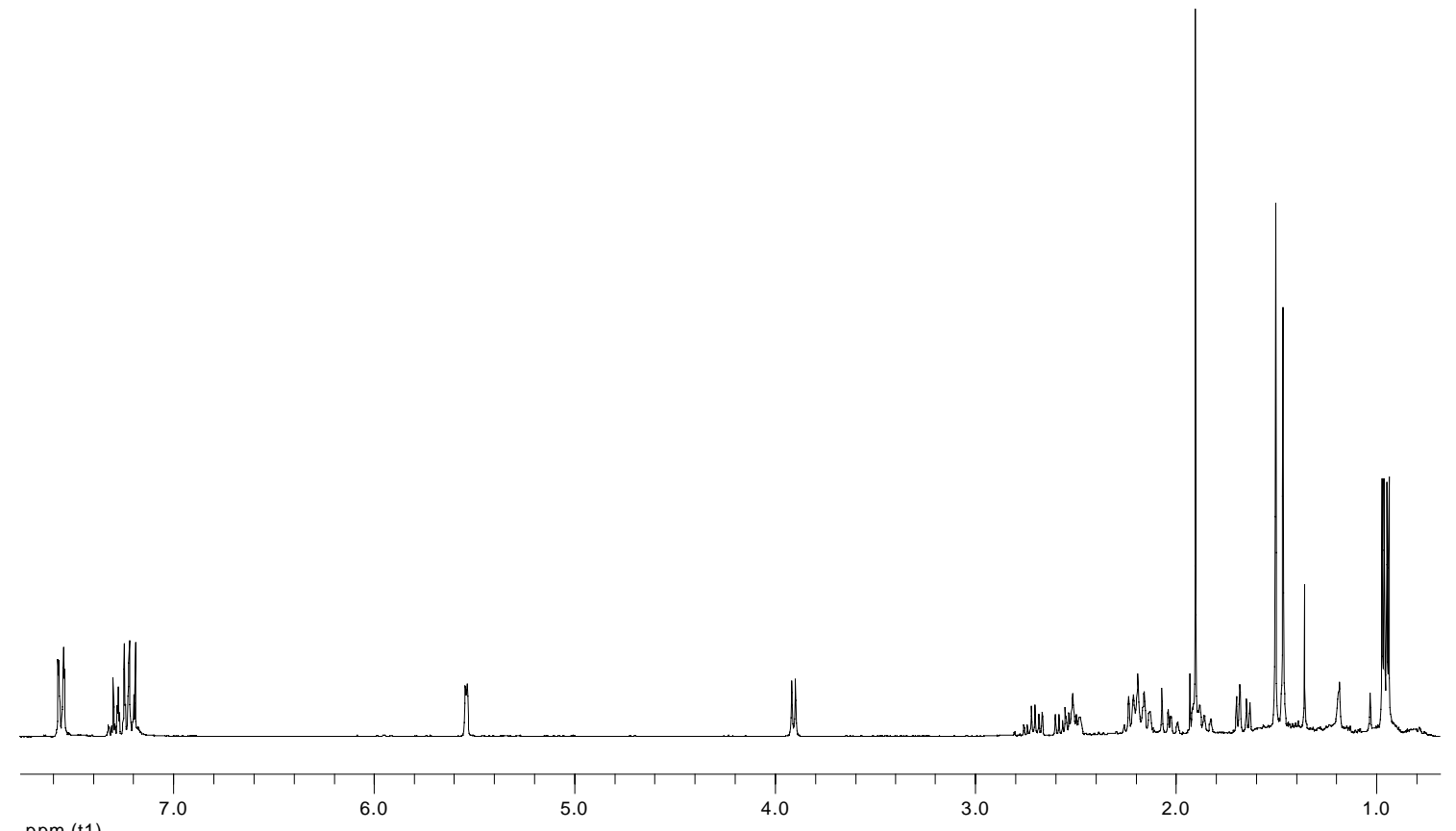

${ }^{13} \mathrm{C}$ NMR $\left(75 \mathrm{MHz}, \mathrm{Cl}_{3} \mathrm{CD}\right)$

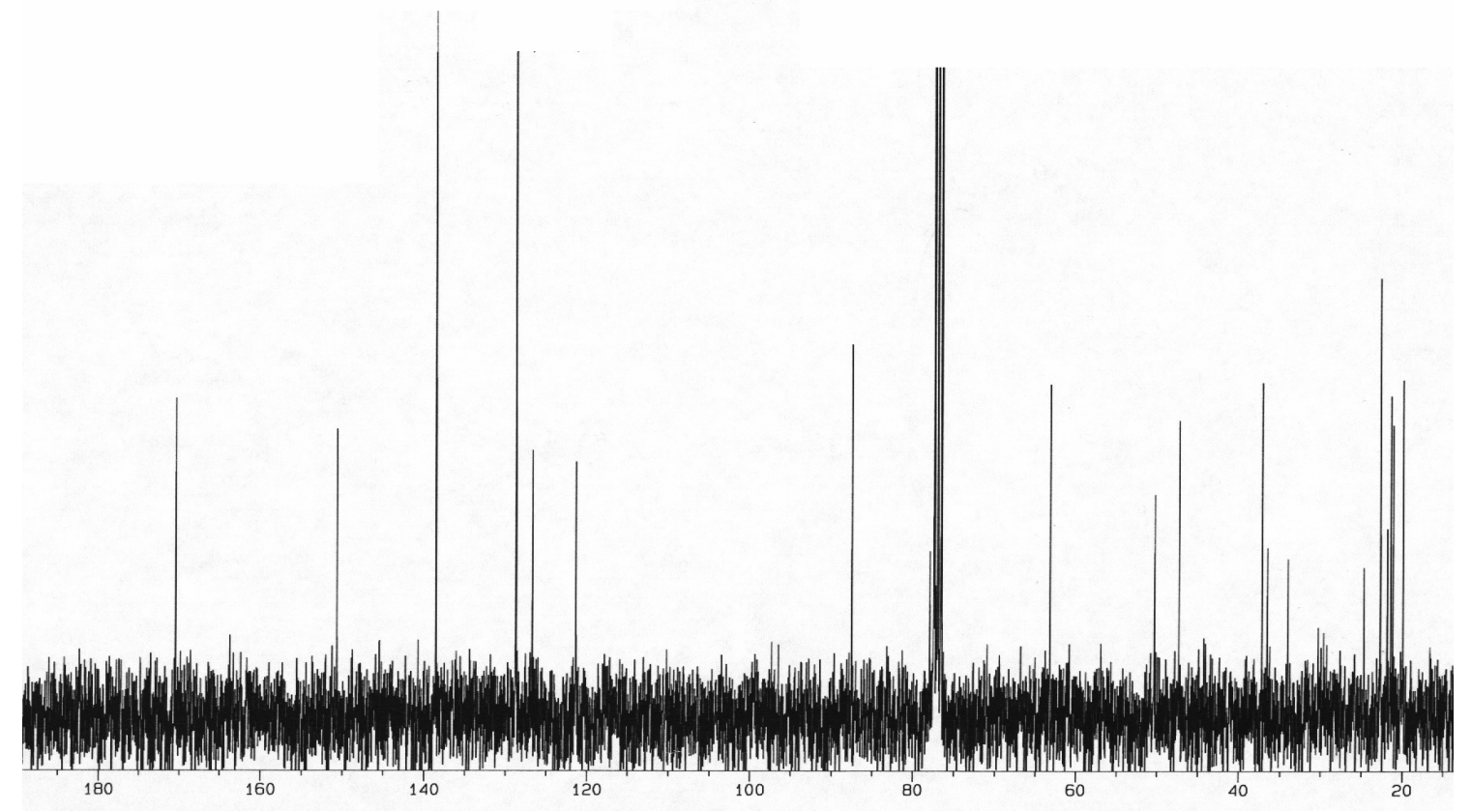

SI- 8 


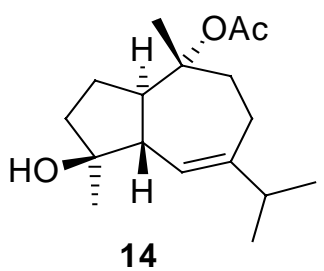

${ }^{1}$ H NMR (400 MHz, $\left.\mathrm{Cl}_{3} \mathrm{CD}\right)$

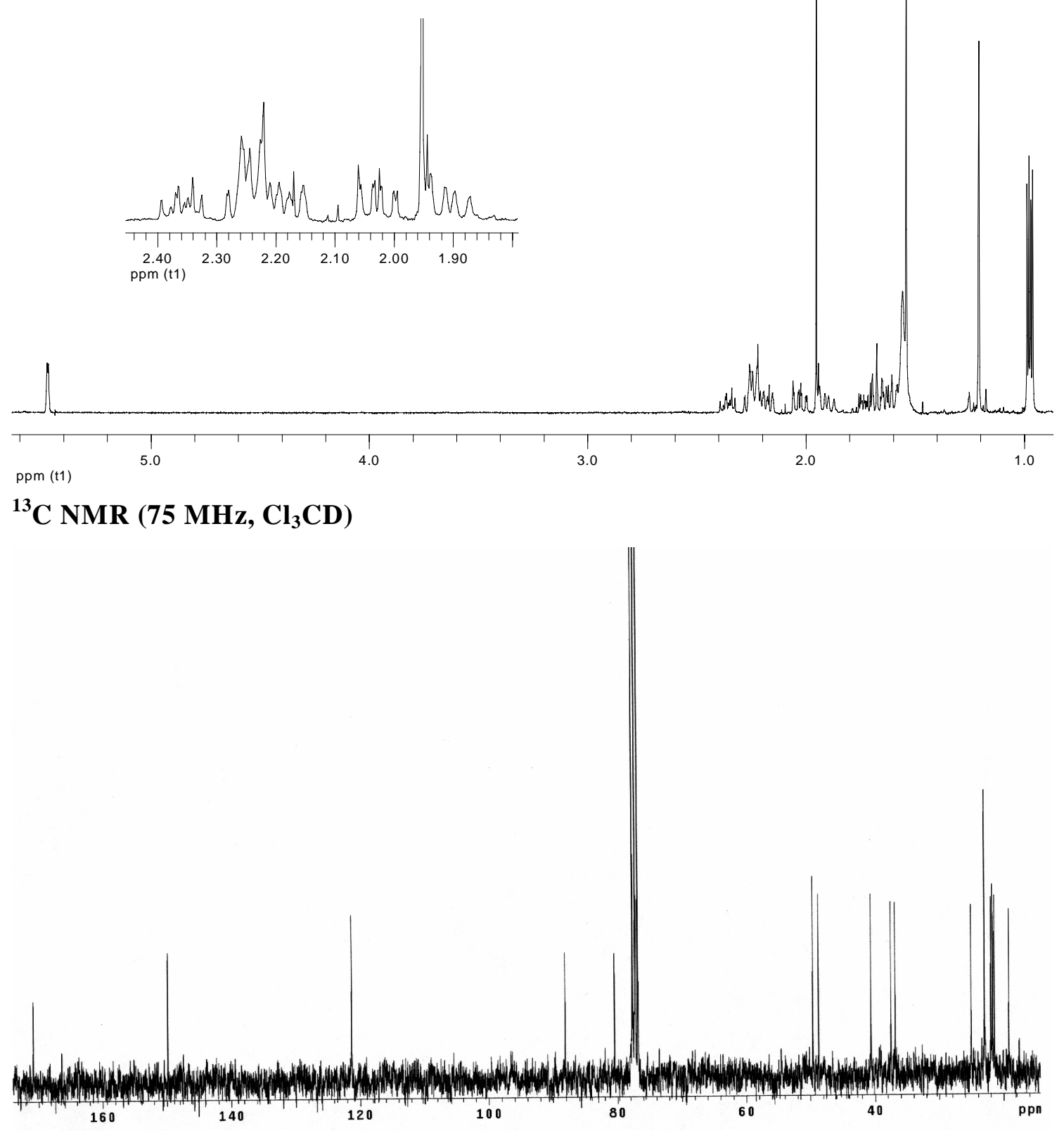

SI- 9 


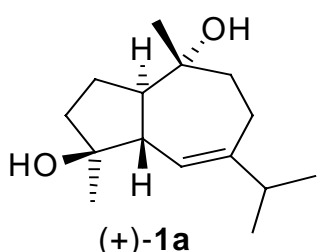

${ }^{1} \mathrm{H}$ NMR (400 MHz, $\left.\mathrm{Cl}_{3} \mathrm{CD}\right)$

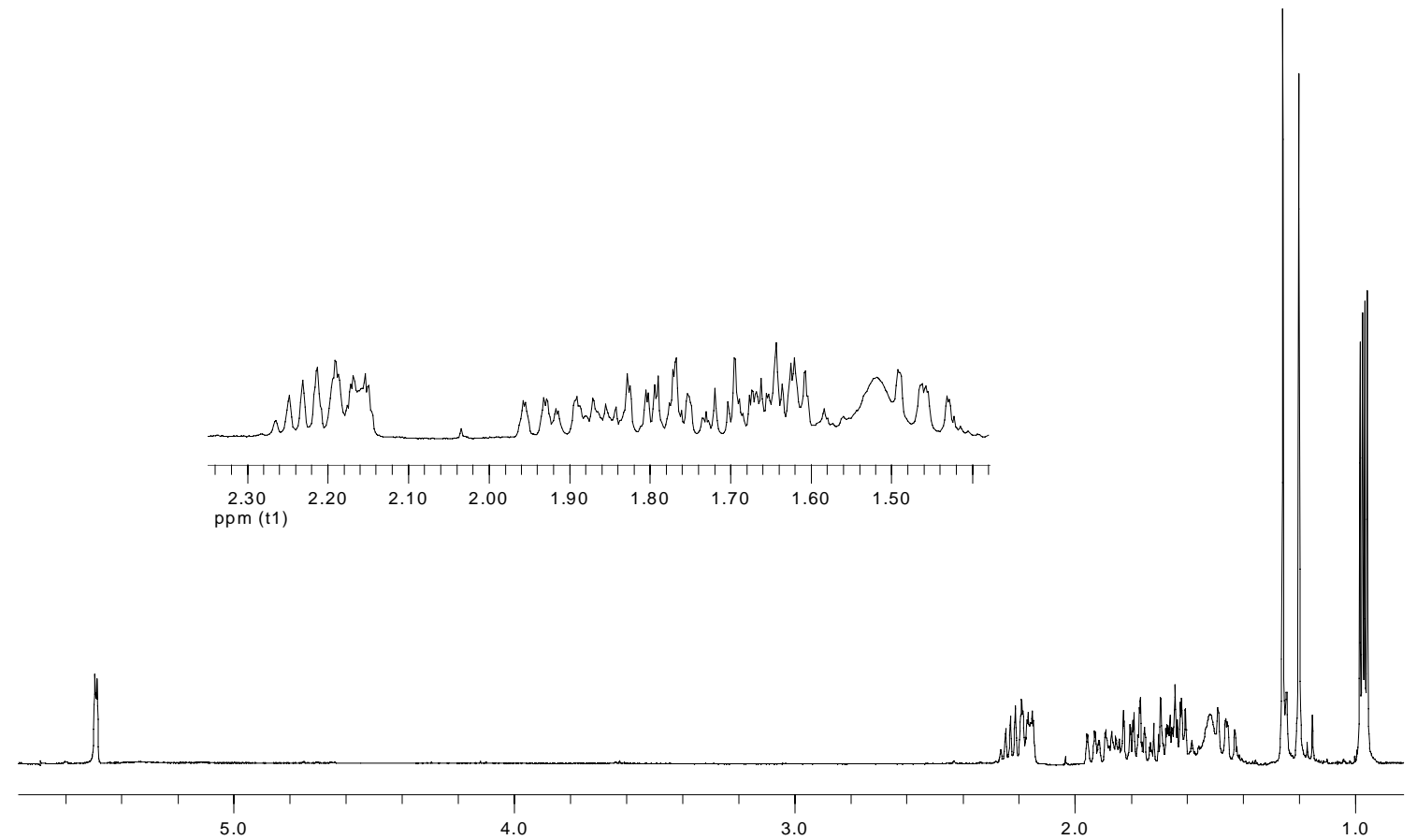

ppm (t1)

${ }^{13} \mathrm{C}$ NMR $\left(75 \mathrm{MHz}, \mathrm{Cl}_{3} \mathrm{CD}\right)$

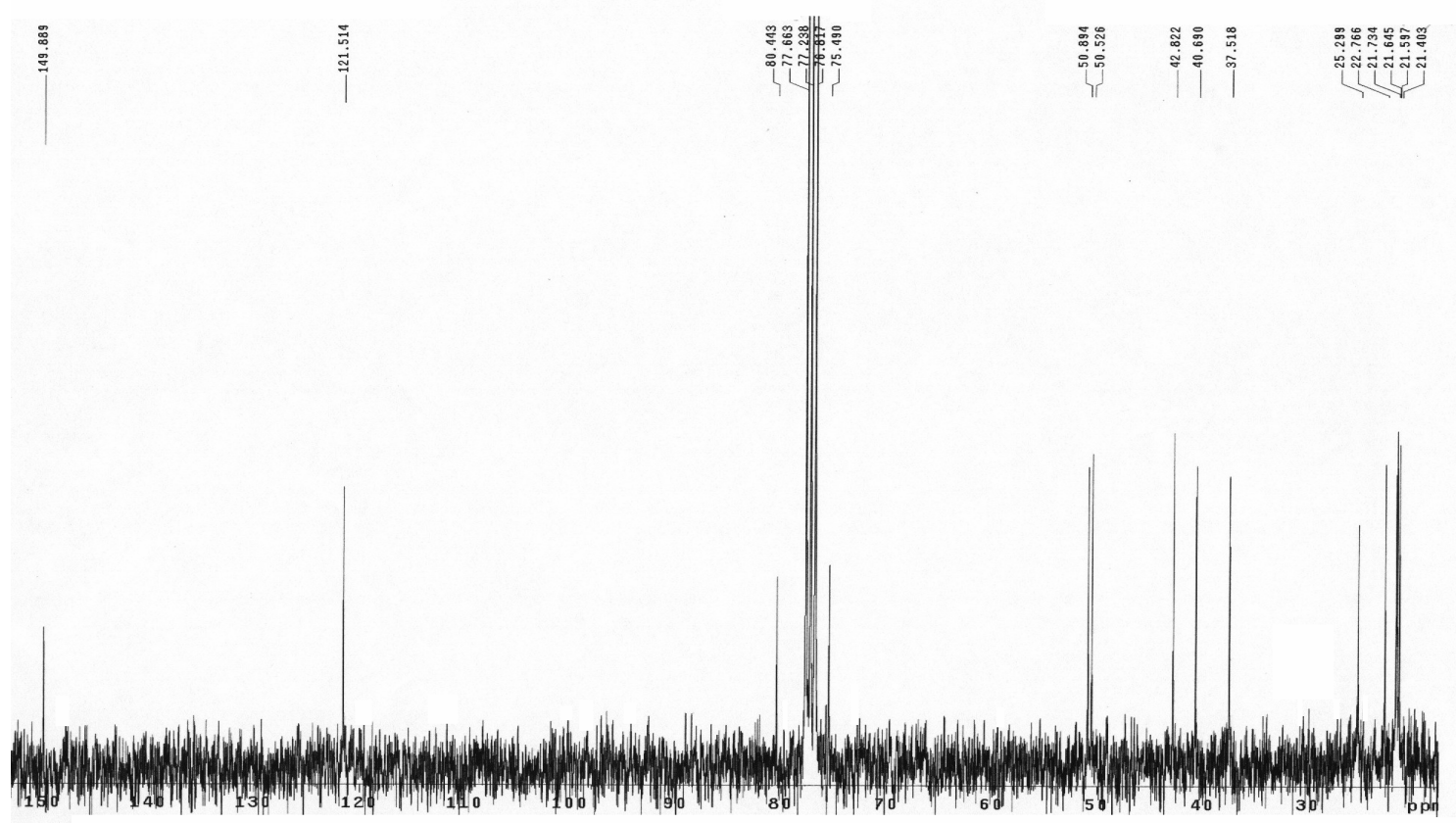




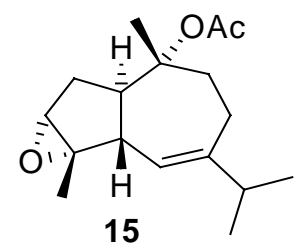

${ }^{1} \mathrm{H}$ NMR (400 MHz, $\left.\mathrm{Cl}_{3} \mathrm{CD}\right)$

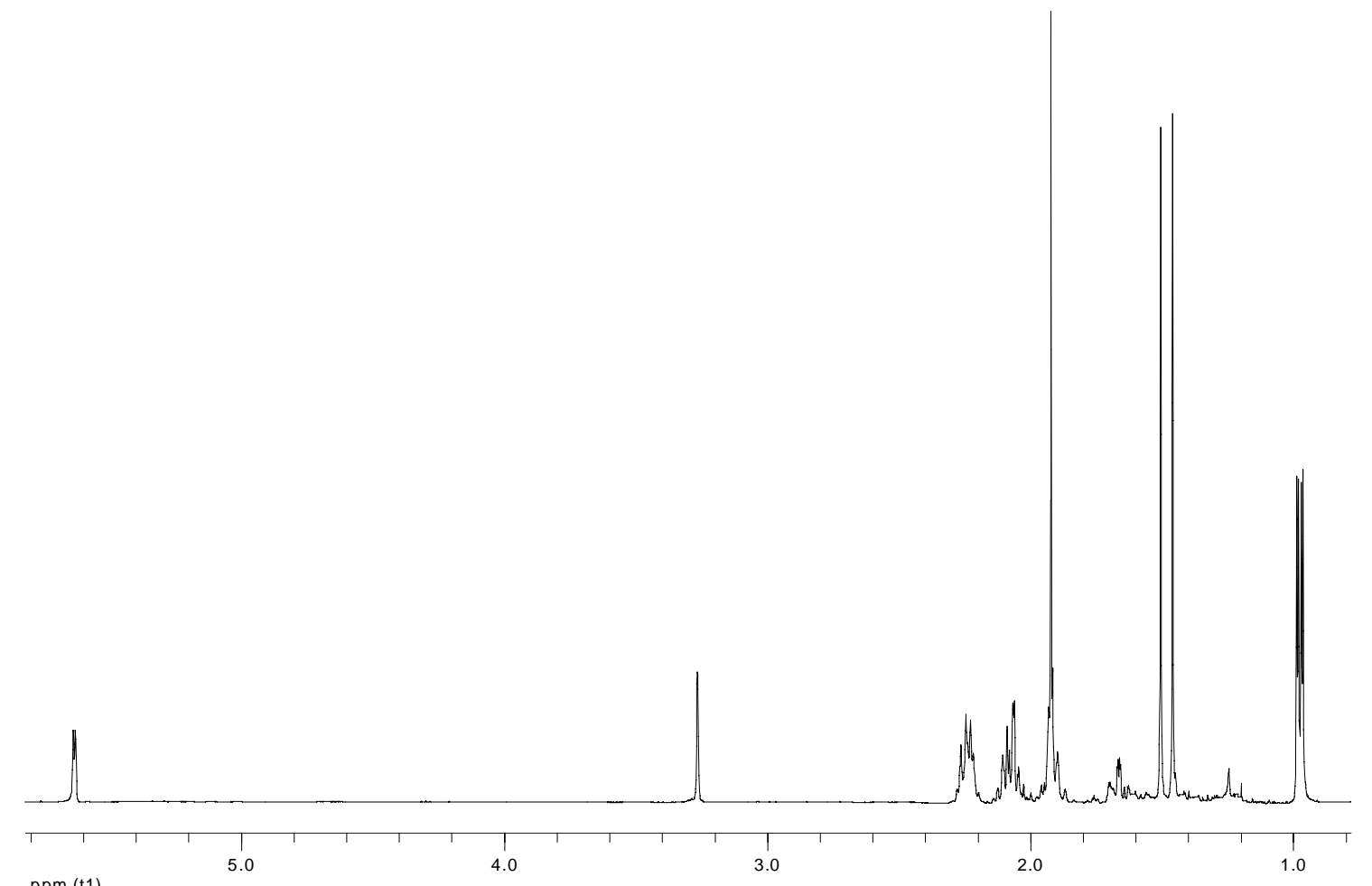

${ }^{13} \mathrm{C}$ NMR (75 MHz, $\left.\mathrm{Cl}_{3} \mathrm{CD}\right)$

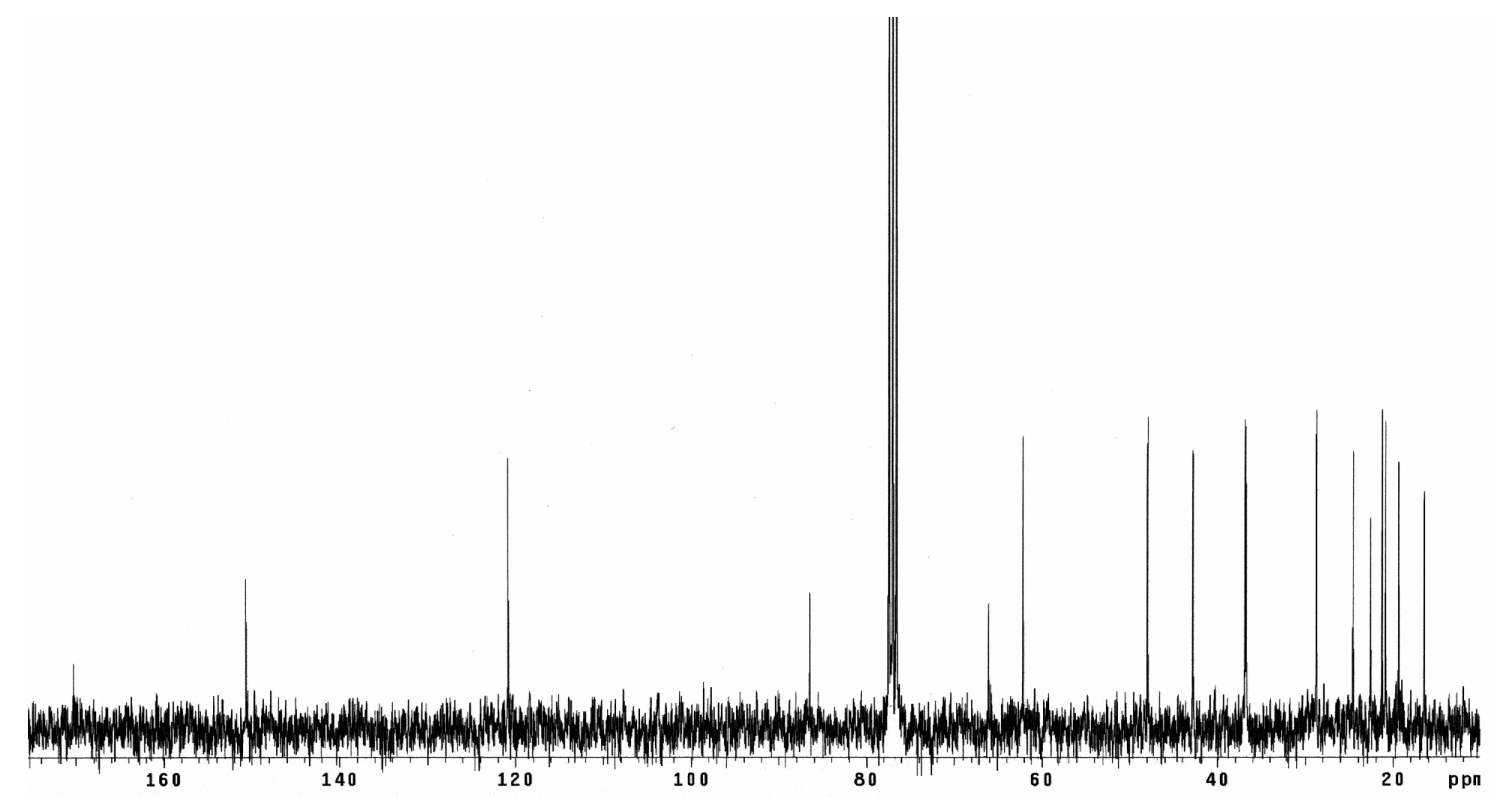

SI- 11 


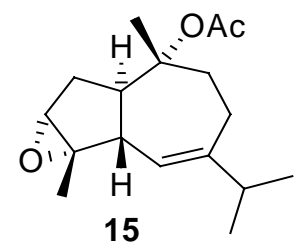

NOE (400 MHz, $\left.\mathrm{Cl}_{3} \mathrm{CD}\right)$
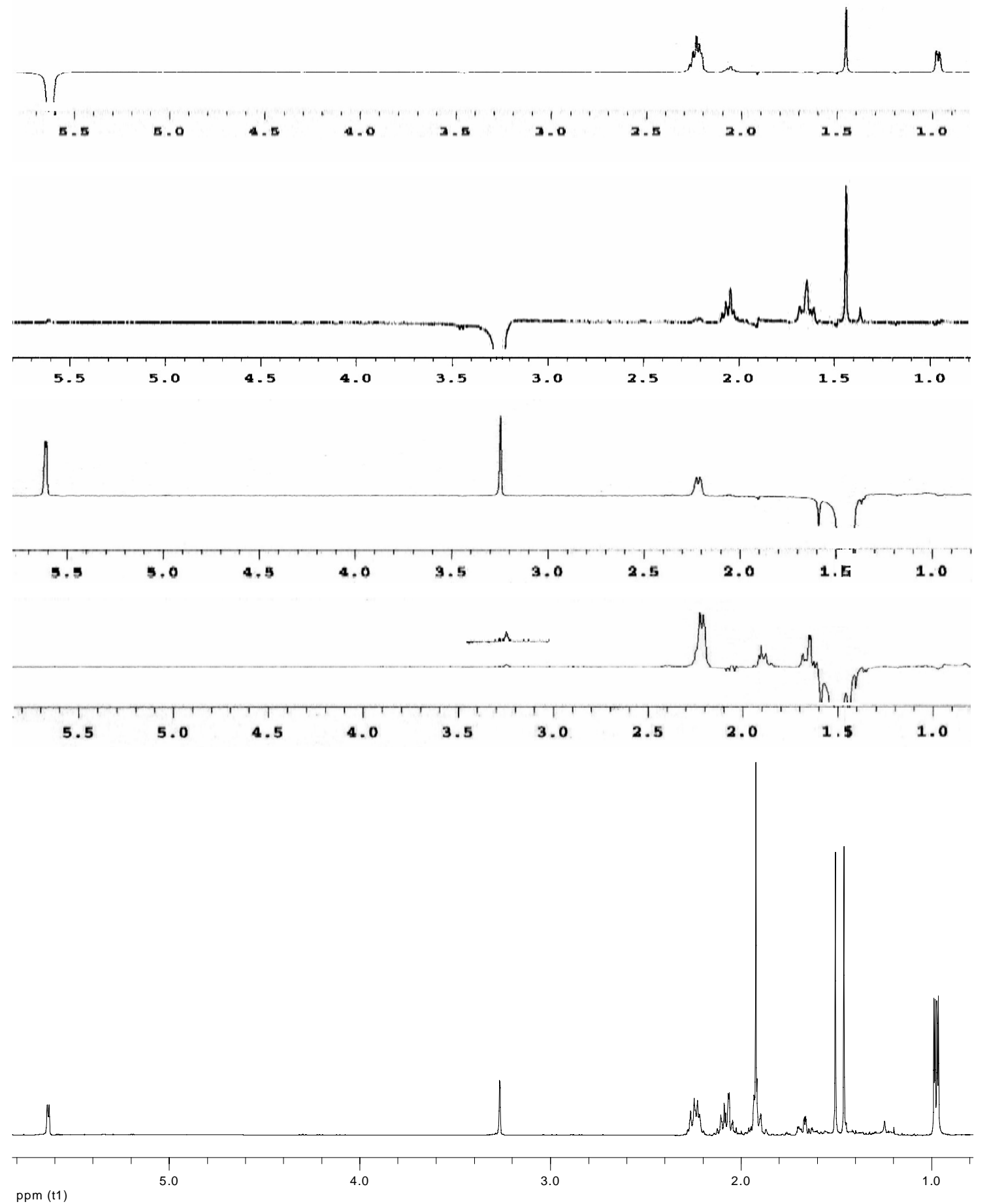


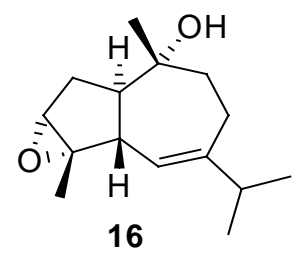

${ }^{1} \mathrm{H}$ NMR (400 MHz, $\left.\mathrm{Cl}_{3} \mathrm{CD}\right)$

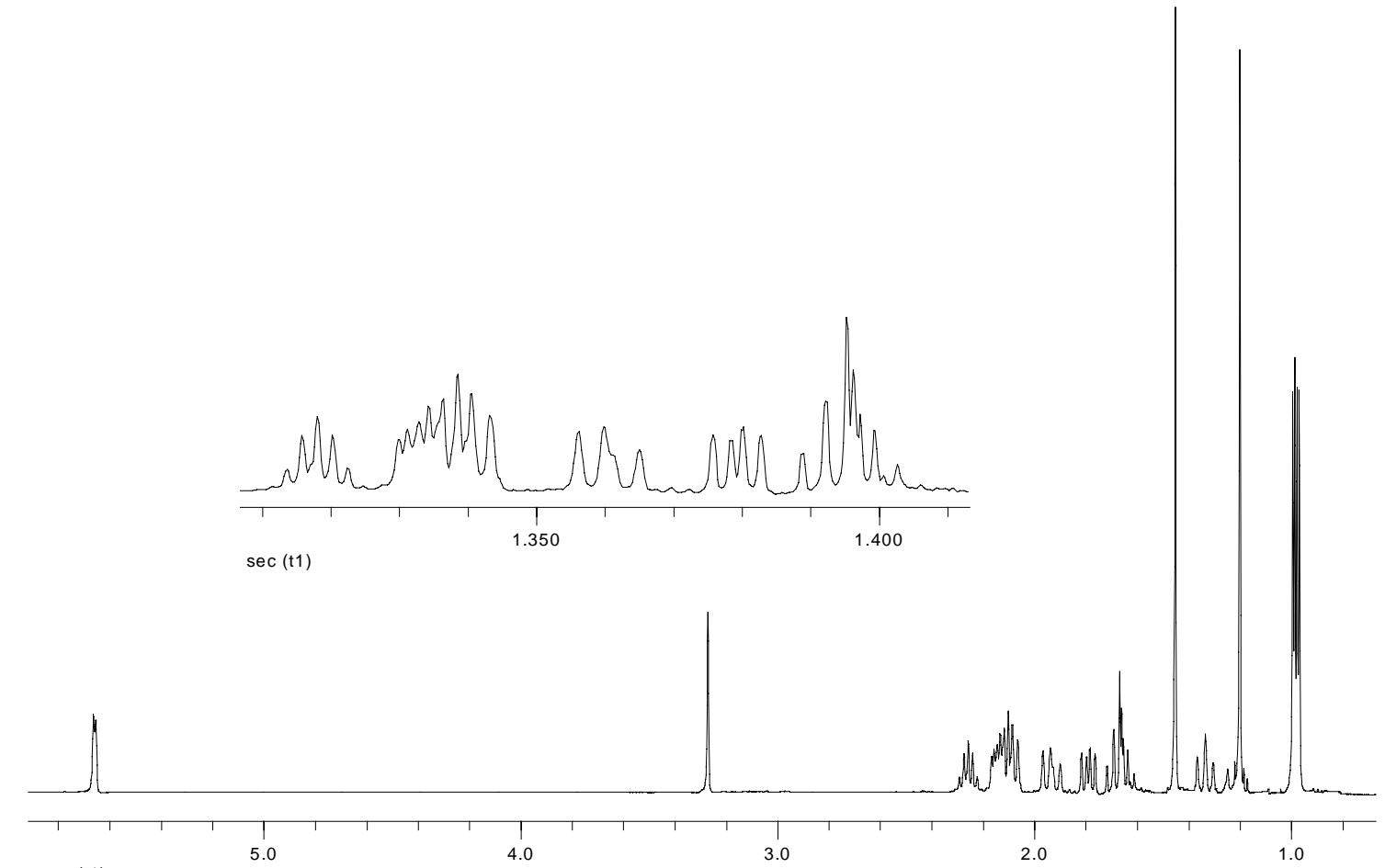

ppm (t1)

${ }^{13} \mathrm{C}$ NMR (75 MHz, $\left.\mathrm{Cl}_{3} \mathrm{CD}\right)$

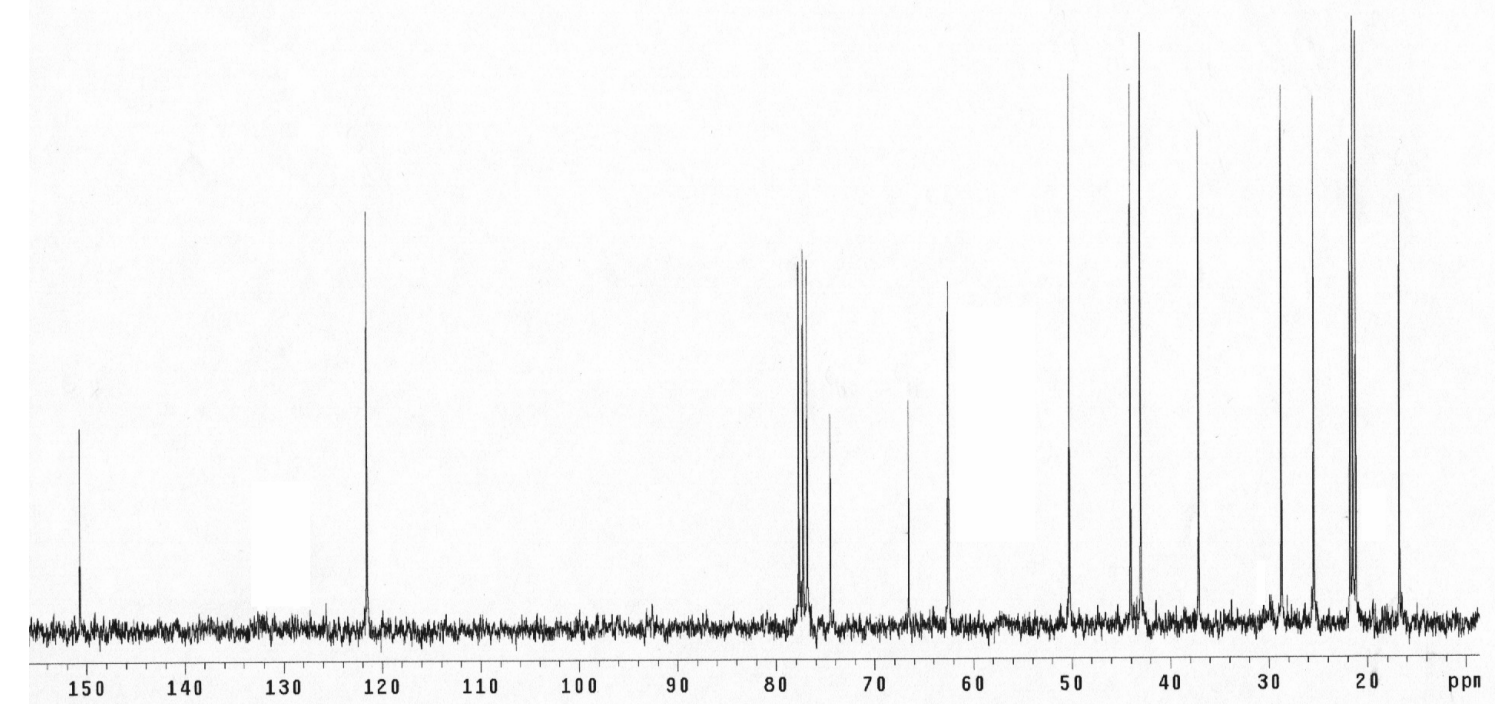

SI- 13 


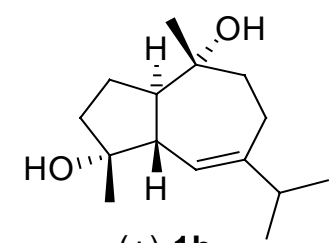

$(+)-\mathbf{1 b}$

\section{${ }^{1} \mathrm{H}$ NMR (400 MHz, $\left.\mathrm{Cl}_{3} \mathrm{CD}\right)$}

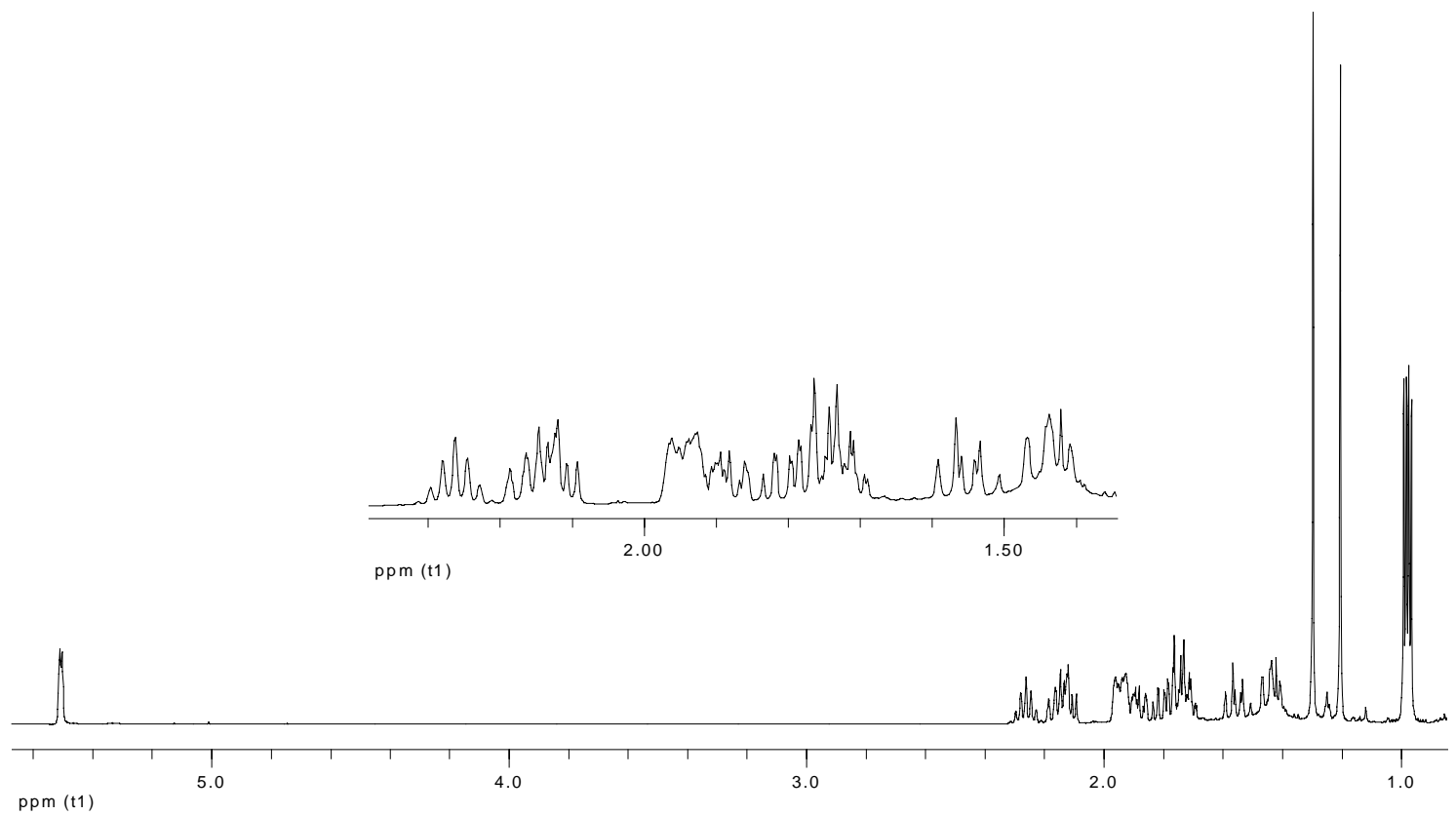

COSY (400 MHz, $\mathrm{Cl}_{3} \mathrm{CD}$ )

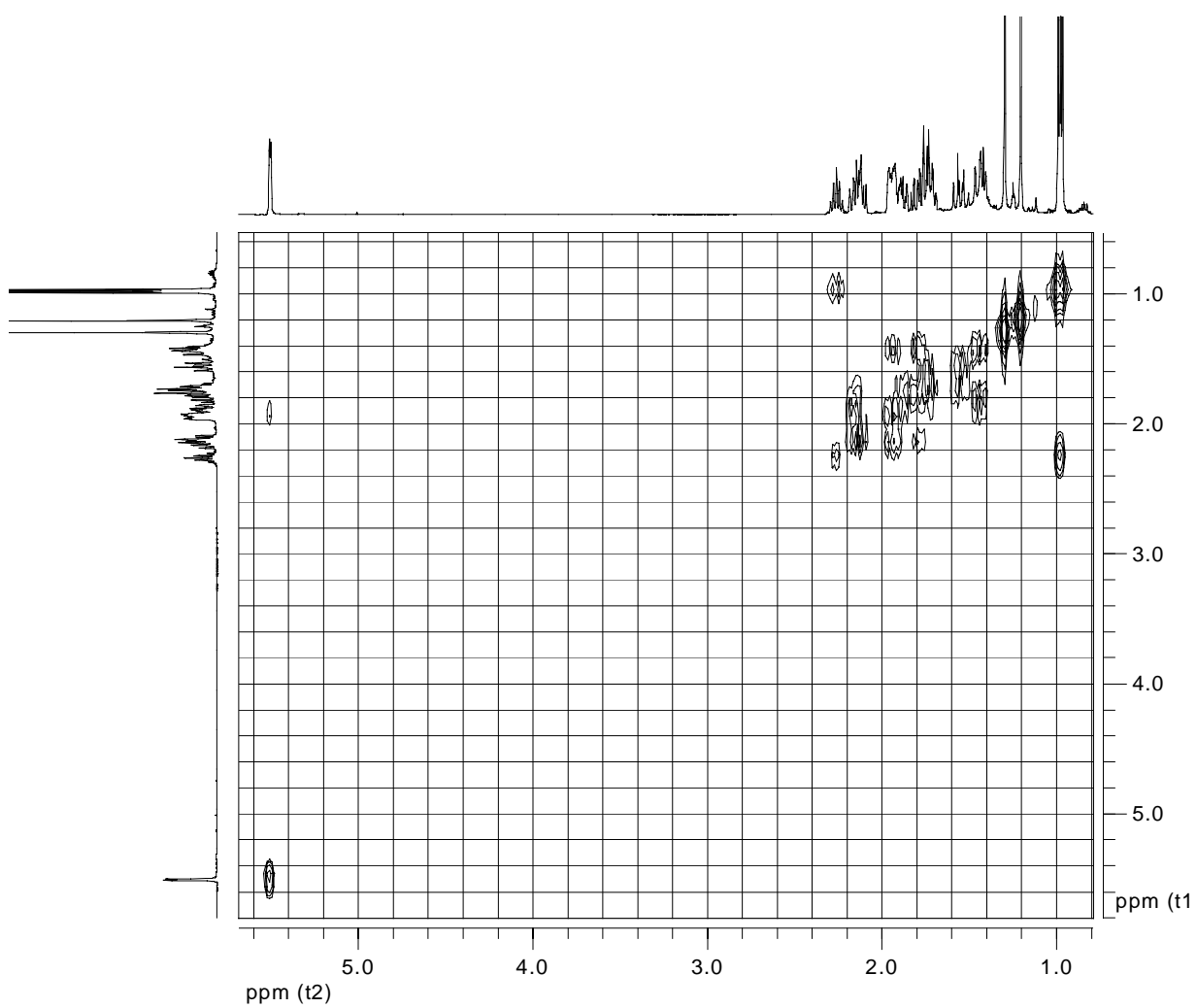

SI- 14 


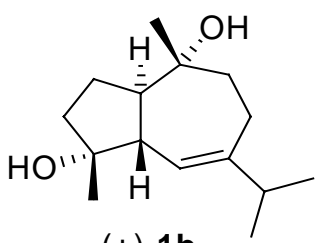

(+)-1b

${ }^{13} \mathrm{C}$ NMR (75 MHz, $\left.\mathrm{Cl}_{3} \mathrm{CD}\right)$
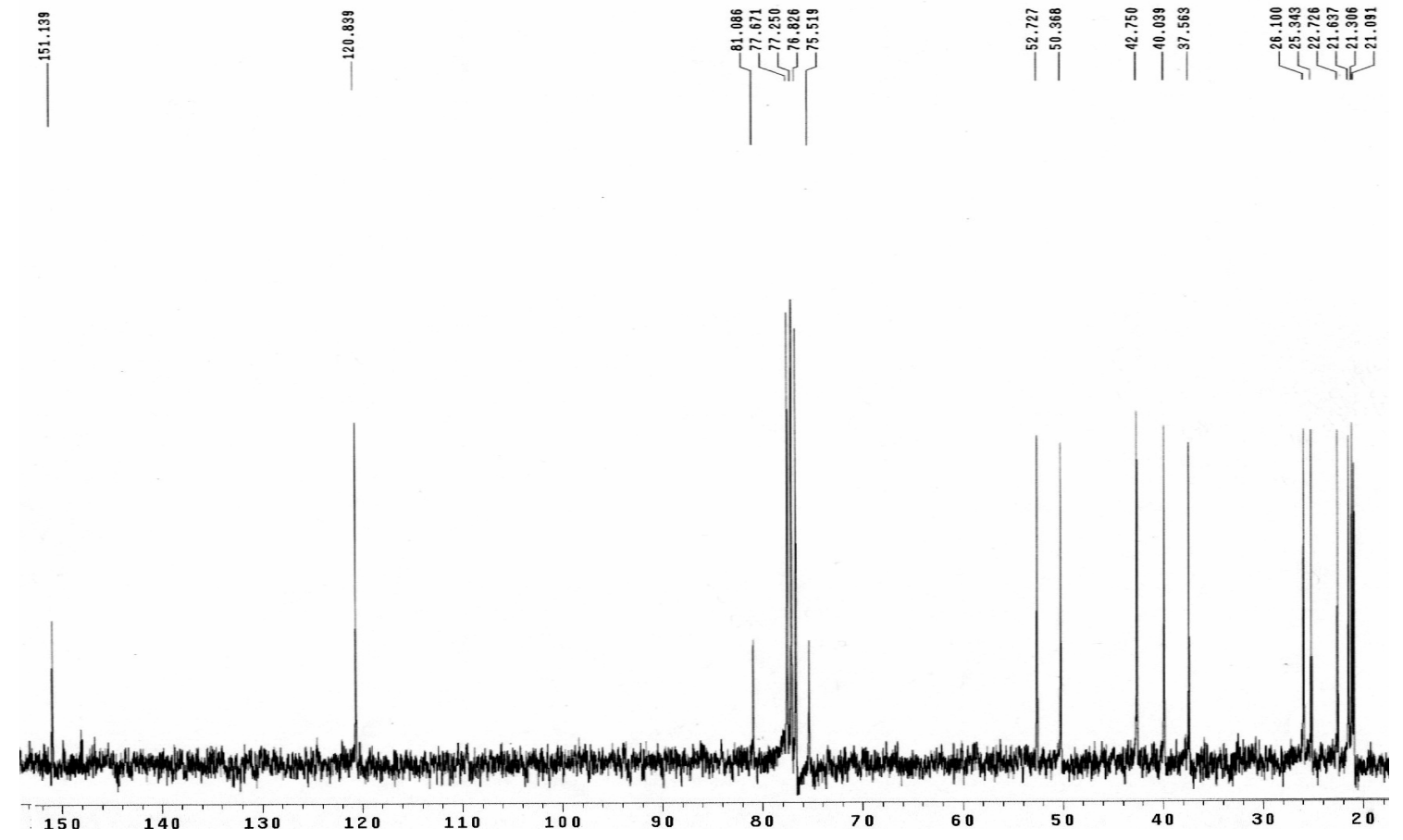

HMQC (400 MHz, $\left.\mathrm{Cl}_{3} \mathrm{CD}\right)$

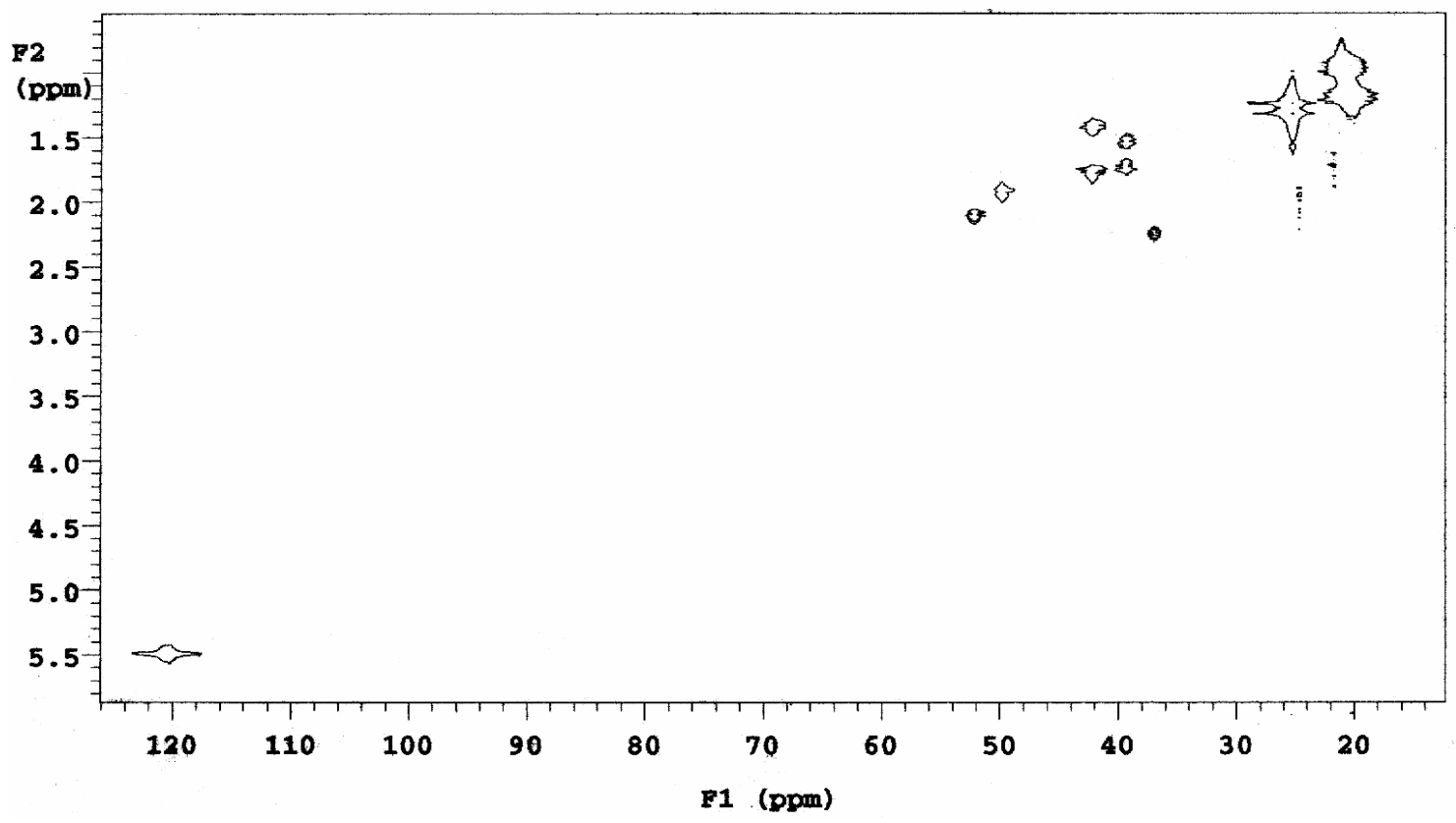

SI- 15 


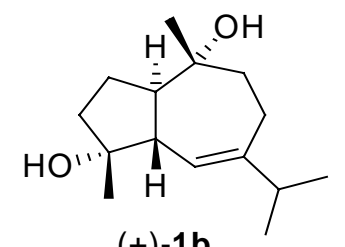

(+)-1b

NOE Experiments (400 $\mathrm{MHz}, \mathrm{Cl}_{3} \mathrm{CD}$ )
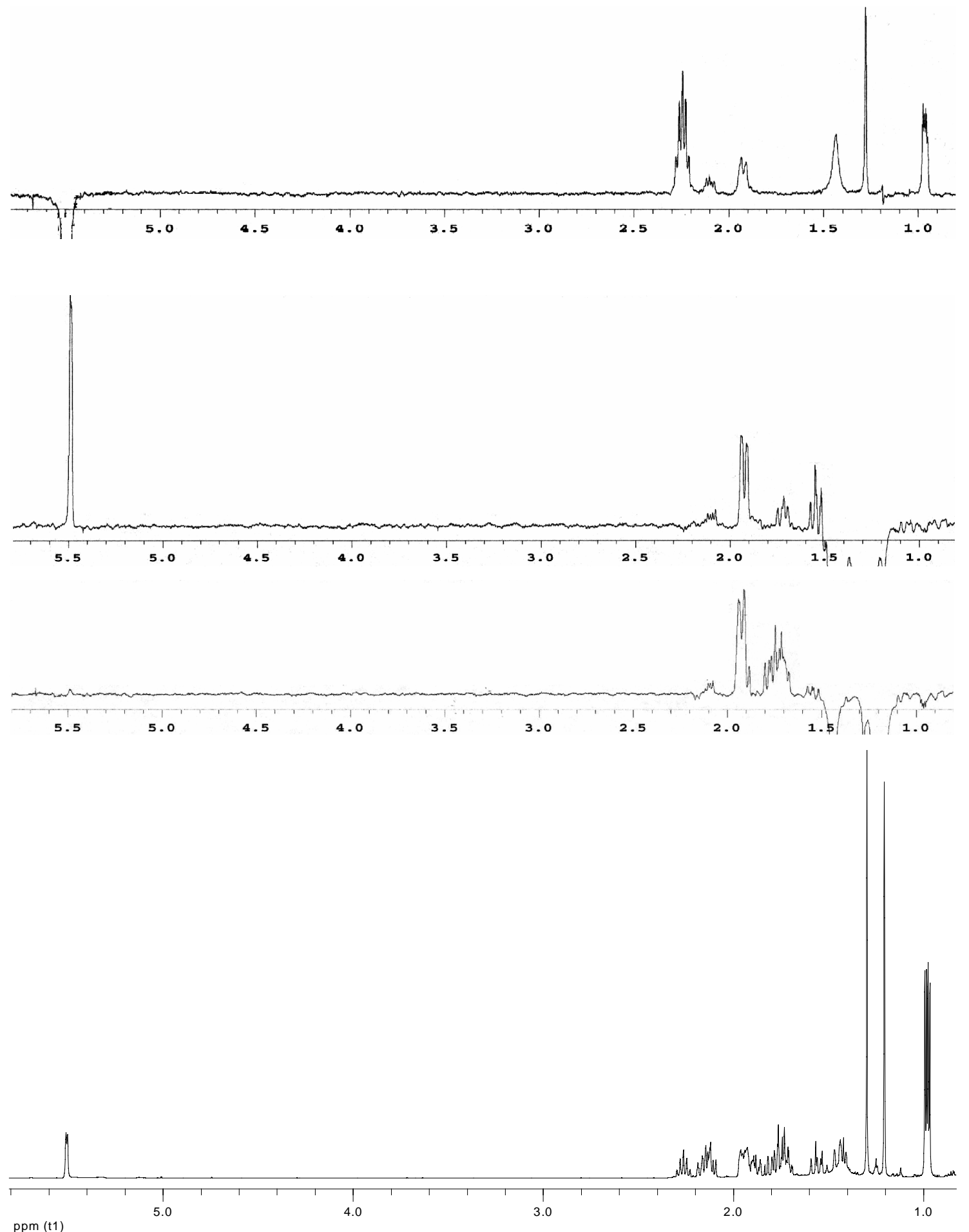